\title{
RADIOEMISSÕES SOLARES TIPO II ASSOCIADAS A FLARES E CMES
}

\section{SOLAR TYPE II RADIO BURSTS ASSOCIATED WITH FLARES AND CMES}

\author{
Rafael Douglas Cunha-Silva ${ }^{1}$ \\ Francisco Carlos Rocha Fernandes ${ }^{2}$ \\ Caius Lucius Selhorst ${ }^{2}$
}

RESUMO: Atribuídas a ondas de choque e a ejeções de plasmoide, as emissões solares tipo II são ondas eletromagnéticas geradas a partir de oscilações do plasma coronal. A origem dos choques associados a essas emissões é ainda uma questão em aberto da física solar. Enquanto alguns trabalhos sugerem os flares solares como seus acionadores, outros fornecem indícios de serem as ejeções de massa coronal (CMEs) sua origem mais provável. Este trabalho apresenta os resultados da análise de duas emissões tipo II, registradas por dois espectrômetros da rede e-CALLISTO (extended-Compound Astronomical Low-cost Low-frequency Instrument for Spectroscopy and Transportable Observatory), os quais operam na faixa de frequências de 45-870 MHz. O primeiro evento, observado em 13 de junho de 2010, às 05:38 UT, apresentou uma taxa de deriva em frequência de -0,2 $\mathrm{MHz} \mathrm{s}^{-1}$, correspondente a uma velocidade de choque de $528 \mathrm{~km} \mathrm{~s}^{-1}$, estando, temporalmente, associado a uma CME lenta $\left(\sim 320 \mathrm{~km} \mathrm{~s}^{-1}\right)$ e a um flare solar em raios- $X$, classe M1.0. O segundo evento, observado em 09 de Agosto de 2011, às 08:02 UT, apresentou uma taxa de deriva em frequência de $-1,4 \mathrm{MHz} \mathrm{s}^{-1}$, correspondente a uma velocidade de choque de $1375 \mathrm{~km} \mathrm{~s}^{-1}$, estando, temporalmente, associado a uma CME tipo halo ( 1610 $\mathrm{km} \mathrm{s}^{-1}$ ) e a um flare solar em raios-X, classe X6.9. Os resultados obtidos, para os parâmetros observacionais das emissões tipo Il e para os parâmetros físicos de suas fontes, são discutidos no contexto de sua relação com seu flares e CMEs associados.

Palavras-chave: radioemissões solares tipo II; flares; CMEs; e-CALLISTO.

ABSTRACT: Solar type II radio bursts are interpreted as signatures of shock waves and plasmoid ejections, being characterized as electromagnetic waves generated by plasma oscillation in the solar corona. Nevertheless, there are long-standing controversies among solar physicists about how such shocks are produced, flares and coronal mass ejections (CMEs) being the most likely drivers. Some observations point to the former, whereas others to the latter. This work analyzes 2 type II bursts recorded by two spectrometers integrating into e-CALLISTO (extended-Compound Astronomical Low-cost Low-frequency Instrument for Spectroscopy and Transportable Observatory), which operates in the frequency range of 45-870 MHz. The first event, observed on June 13, 2010 at 05:38 UT, had a frequency drift rate of $-0.2 \mathrm{MHz} \mathrm{s}^{-1}$ and a shock speed of $528 \mathrm{~km} \mathrm{~s}^{-1}$, and it was temporally associated with a slow CME ( 320 $\left.\mathrm{km} \mathrm{s}^{-1}\right)$ and an M1.0 flare. The second event, observed on August 9, 2011 at 08:02 UT, had a frequency drift rate of $-1.4 \mathrm{MHz} \mathrm{s}^{-1}$ and a shock speed of $1375 \mathrm{~km} \mathrm{~s}^{-1}$, and it was temporally associated with a halo CME ( 1610 $\left.\mathrm{km} \mathrm{s}^{-1}\right)$ and with an X6.9 flare. The observational parameters of the type II bursts and the physical parameters of their sources are discussed in the context of their associated flares and CMEs.

Keywords: solar type II radio bursts; flares; CMEs; e-CALLISTO.

\footnotetext{
1 Doutorando em Física e Astronomia - Universidade do Vale do Paraíba - Univap / Instituto de Pesquisa e Desenvolvimento IP\&D. E-mail: rfldoug@gmail.com.

2 Doutor em Astrofísica - INPE e Docente da UNIVAP / IP\&D - Laboratório de Física e Astronomia. E-mails: guga@univap.br; caius@univap.br.
} 


\section{INTRODUÇÃO}

As emissões solares tipo II são atribuídas a ondas de choque e ejeções de plasmoide (RAMESH et al., 2010), caracterizam-se como ondas eletromagnéticas que, ao serem geradas a partir de oscilações de plasma (NELSON; MELROSE, 1985), escapam das imediações do choque (CLIVER et al., 2005). Entre suas características, as emissões tipo II apresentam duração de alguns minutos ( 115 min.), baixa frequência de corte ( 0,1-60 $\mathrm{MHz}$ ), e uma taxa de deriva em frequência lenta $\left(\sim 0,1-1,5 \mathrm{MHz} \mathrm{s}^{-1}\right)$, rumo a frequências mais baixas (AGUILAR-RODRIGUEZ et al., 2005; CUNHA-SILVA; FERNANDES; SELHORST, 2013).

As emissões tipo II são geralmente acompanhantes de flares solares impulsivos e precursoras de CMEs (VOURLIDAS, 2004). Sua taxa de deriva em frequência negativa implica um movimento ascendente de matéria com velocidades da ordem de 200 a $2000 \mathrm{~km} \mathrm{~s}^{-1}$ (ASCHWANDEN, 2005), o qual pode estar associado a uma CME ou à liberação súbita de energia durante um flare solar (SHANMUGARAJU; MOON; VRSNAK, 2009).

Embora a associação entre ondas de choque e emissões tipo II esteja bem evidenciada, os detalhes da estrutura do choque e de seus agentes acionadores necessitam ser esclarecidos (VRSNAK; CLIVER, 2008; PRAKASH et al., 2010).

Este trabalho apresenta os resultados da análise de duas emissões tipo II, registradas por dois espectrômetros da rede e-CALLISTO (BENZ et al., 2009), a qual é constituída, atualmente, por mais de 50 espectrômetros em operação ao redor do mundo, com vistas a realizar o monitoramento contínuo da atividade solar na faixa de frequências de $45-870 \mathrm{MHz}$.

\section{OBSERVAÇÕES E ANÁLISE}

O primeiro evento tipo II investigado foi observado em 13 de junho de 2010, às 05:38 UT, pelo espectrômetro da rede eCALLISTO em Ooty (Índia), enquanto o segundo evento foi observado em 09 de Agosto de 2011, às 08:02 UT, pelo espectrômetro da rede e-CALLISTO, em Bleien (Suíça). As Figuras 1 e 2 apresentam os espectros dinâmicos dos dois eventos tipo II investigados e seus respectivos máximos de intensidade, identificados por via dos perfis espectrais das emissões. 

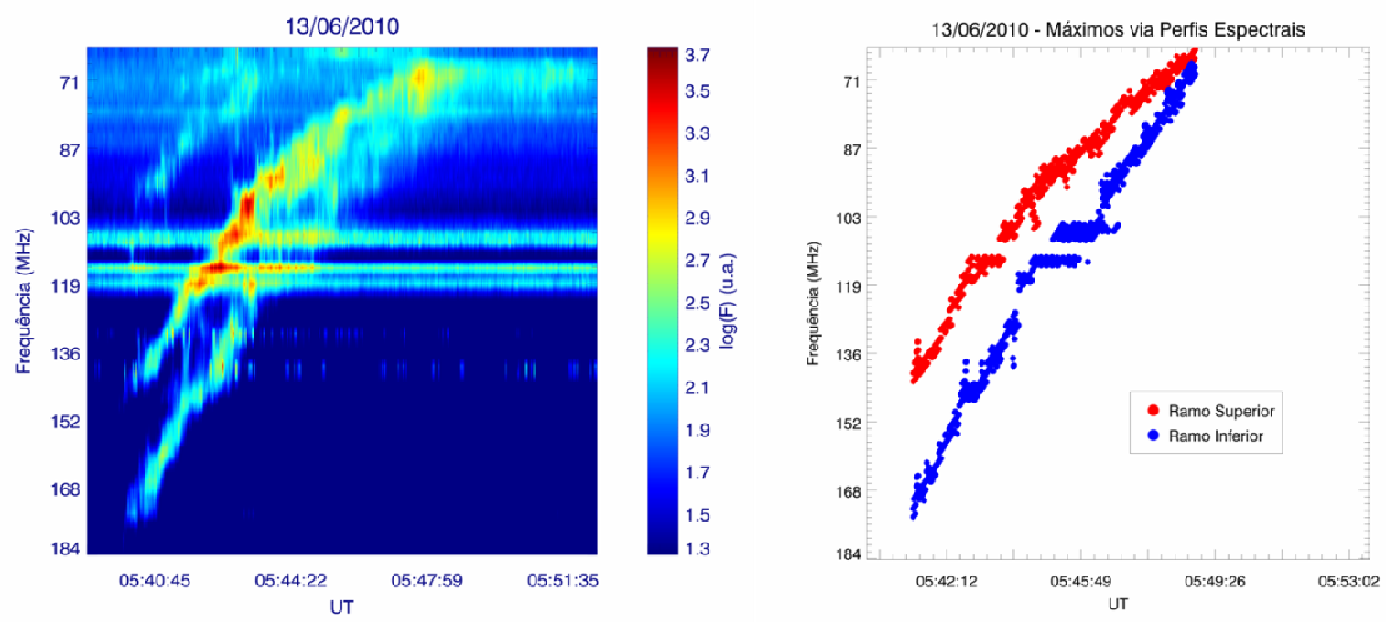

Figura 1 - Espectro dinâmico e máximos de intensidade de emissão tipo II, registrada pelo OOTY, em 13 de junho de 2010, às 05:38 UT.
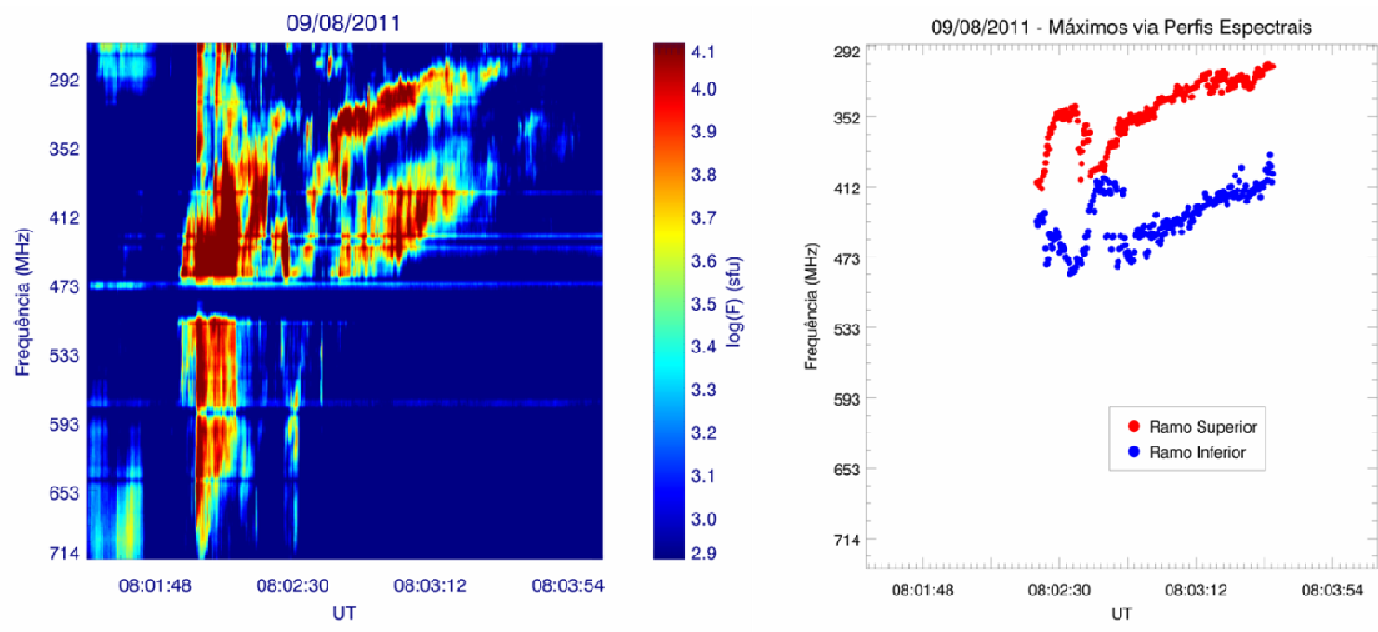

Figura 2 - Espectro dinâmico e máximos de intensidade de emissão tipo II, registrada pelo BLEN7M, em 09 de agosto de 2011, às 08:02 UT.

\section{RESULTADOS}

A partir dos parâmetros observacionais das emissões, tais como a taxa de deriva em frequência, a largura de banda instantânea e a divisão de banda instantânea, foram estimados, para suas fontes, os seguintes parâmetros físicos: densidade eletrônica; intensidade do campo magnético; velocidade do choque; velocidade de Alfvén; parâmetro beta do plasma; e temperatura. A Figura 3 apresenta a distribuição desses parâmetros com relação às frequências inicial, central e final. 


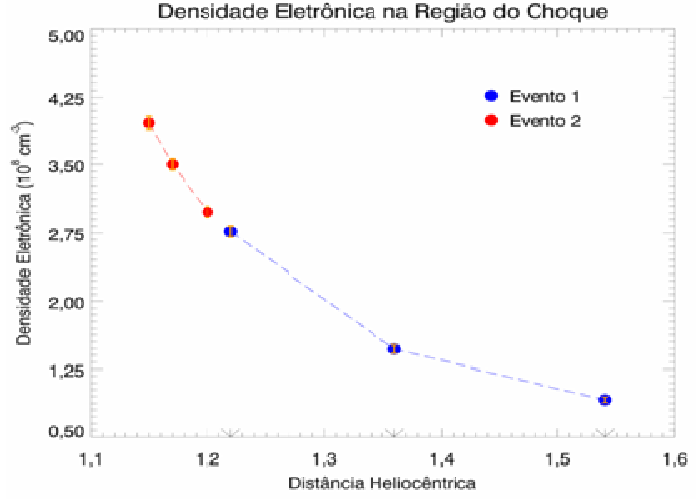

(a)

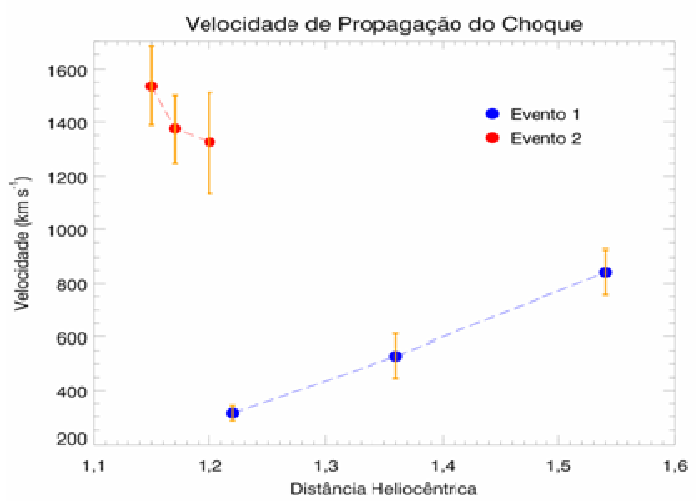

(c)

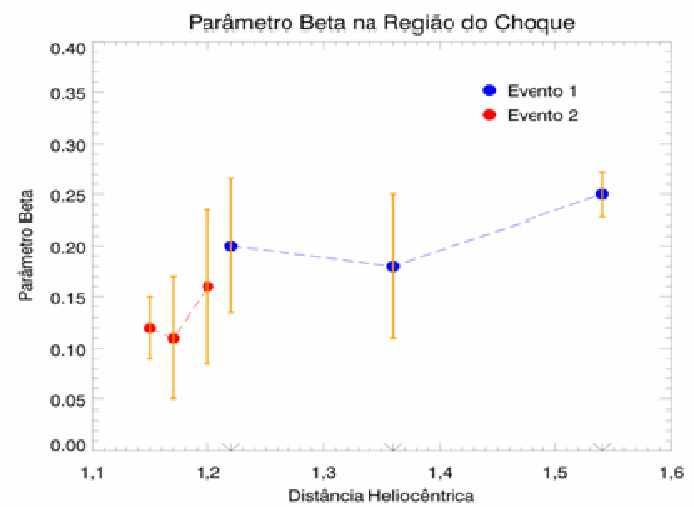

(e)

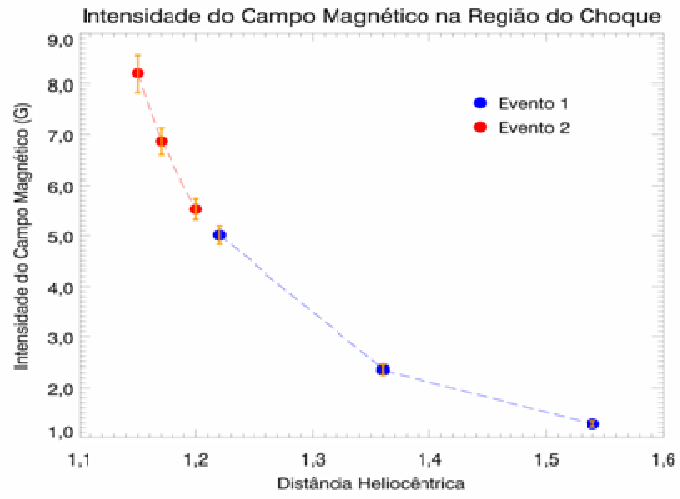

(b)

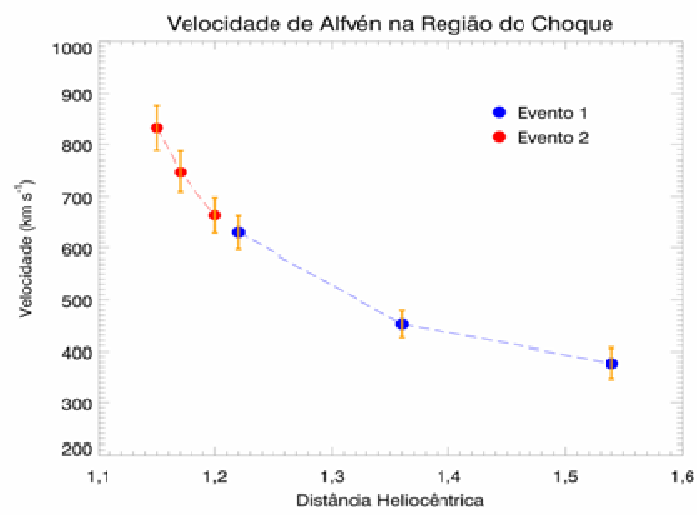

(d)

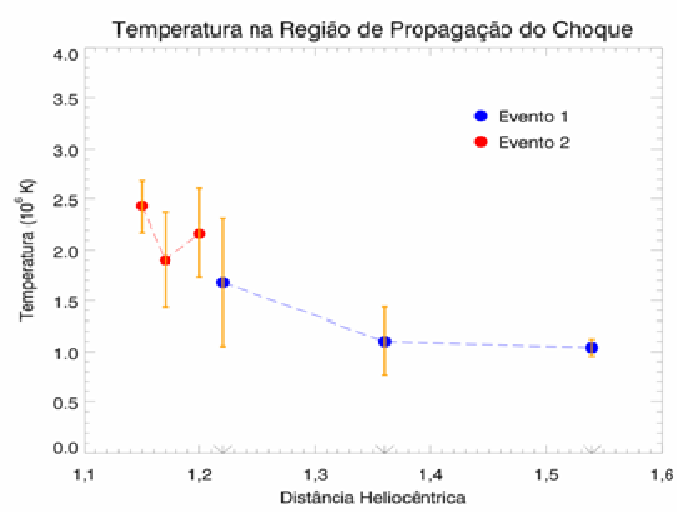

(f)

Figura 3 - (a) Densidade eletrônica, (b) intensidade do campo magnético, (c) velocidade do choque, (d) velocidade de Alfvén, (e) parâmetro beta do plasma, e (f) temperatura, referentes às frequências inicial, central e final.

A Figura 4 apresenta uma comparação II, de suas CMEs associadas e do pico de entre os instantes de início dos eventos tipo seus flares. 

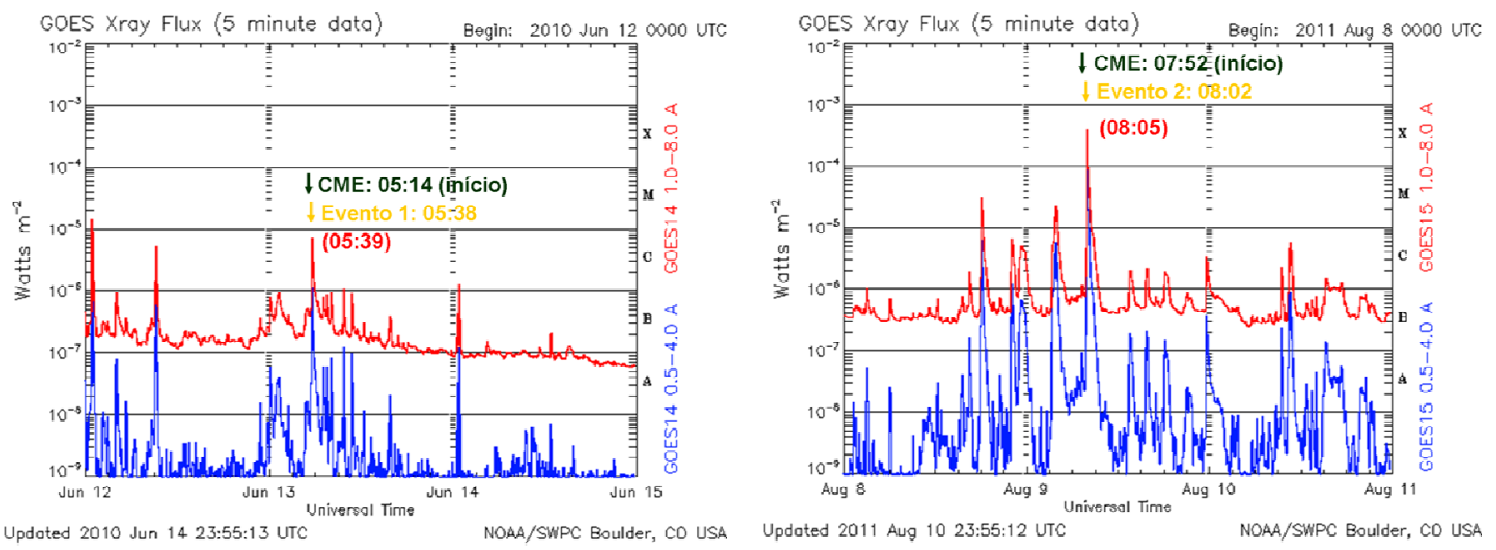

Figura 4 - Comparação entre os instantes de início dos eventos tipo II, de suas CMEs associadas e do pico de seus flares.

\section{CONCLUSÕES}

O presente estudo proporcionou uma investigação das propriedades físicas das fontes das emissões tipo II analisadas, cuja interpretação, no contexto de seus flares e CMEs associados, permitiu as seguintes constatações:

- As velocidades dos choques associados aos eventos são compatíveis com as velocidades de suas CMEs associadas;

- O flare mais intenso, associado ao Evento 2, esteve também associado a uma emissão tipo III;

- A fase impulsiva dos flares associados aos eventos ocorreu durante 0 início da fase de aceleração das CMEs associadas, conforme observado por Zhang et al. (2001);

- Houve fortes indícios de velocidades subalfvênicas para as CMEs associadas aos eventos antes do início de seus flares associados, o que reforça o argumento apresentado por Cliver et al. (2005).

\section{AGRADECIMENTOS}

R. D. Cunha-Silva agradece a bolsa de Doutorado - Processo no 2012/00009-5, FAPESP e à Fundação Valeparaibana de Ensino (FVE), pela isenção de mensalidade. F. C. R. Fernandes agradece ao CNPq, pela Bolsa de Produtividade em Pesquisa (Proc. 308755/2012-0). Os autores agradecem ao grupo de pesquisadores e estudantes da rede e-CALLISTO, pelos dados observacionais solares.

\section{REFERÊNCIAS}

AGUILAR-RODRIGUEZ, E. et al. A Study of the Drift Rate of Type II Radio Bursts at Different wavelengths. In: In: FLECK, B.; ZURBUCHEN, T. H.; LACOSTE, $H$. PROCEEDINGS OF THE SOLAR WIND $11 /$ SOHO 16, "CONNECTING SUN AND HELIOSPHERE", 2005, Whistler, Canada. Proceedings... Washington: NASA, 2005. p. 393.

ASCHWANDEN, M. J. Physics of the Solar Corona: an introduction with problems and solutions. $2^{\text {nd }}$ ed. Chichester, UK: Springer and Praxis Publishing., 2005.

BENZ, A. O. et al. A World-Wide Net of Solar 
Radio Spectrometers: e-CALLISTO. Earth Moon Planet, v. 104, p. 277-285, 2009.

CLIVER, E. W. et al. Coronal Shocks of November 1997 Revisited: The CME-Type II Timing Problem. Solar Physics, v. 225, p. 105-139, 2005.

CUNHA-SILVA, R. D.; FERNANDES, F. C. R.; SELHORST, C. L. Solar type II radio bursts recorded by CALLISTO-BR. Solar Physics, 2013. (Submitted)

NELSON, G. J.; MELROSE, D. B. Type II bursts. In: MCLEAN, D. J.; LABRUM, N.R. (eds.). Solar Radiophysics: studies of emission from the Sun at metre wavelengths. Cambridge: Cambridge University Press, 1985.

PRAKASH, O. et al. Type-Il Bursts in Meter and Deca-Hectometer Wavelengths and Their Relation to Flares and CMEs: II. Solar Physics, v. 266, p. 135-147, 2010.

RAMESH, R.; KATHIRAVAN, C.; KARTHA,
S. S.; GOPALSWAMY, N. Radiohelio- graph Observations of Metric Type II Bursts and the Kinematics of Coronal Mass Ejections. Astrophysical Journal, v. 712 , n. 1, p. 188193, 2010.

SHANMUGARAJU, A.; MOON, Y.-J.; VRSNAK, B. Type II Radio Bursts with High and Low Starting Frequencies. Solar Physics, v. 254, p. 297-310, 2009.

VOURLIDAS, A. Radio observations of coronal mass ejections. In: GARY, D. E.; KELLER, C. U. (eds.). Solar and Space Weather Radiophysics: current status and future developments. Dordrecht: Kluwer Academic Publishers, 2004.

VRSNAK, B.; CLIVER, E. W. Origin of Coronal Shock Waves. Invited Review. Solar Physics, v. 253, n. 1-2, p. 215-235, 2008.

ZHANG, J. et al. On the temporal relationship between coronal mass ejections and flares. Astrophysical Journal, v. 559, n. 1, p. 452-462, 2001. 